\title{
Medición financiera de la gestión de la responsabilidad social
}

\author{
Pedro P. Franco Concha \\ Magister en Gestión Ambiental para el Desarrollo Sostenible por La Pontificia Universidad Javeriana, \\ Bogotá, Colombia. Master in Business Administration por el Kogod College Business School, The \\ American University. Profesor, Consultor e Investigador. Universidad del Pacífico, Lima Perú.
}

\author{
Any Chan Ng \\ Noelia León Ramos \\ Christian Mendoza Cotrina
}

\section{Resumen}

La responsabilidad social es un tema que ha despertado el interés del sector empresarial en la búsqueda del desarrollo sostenible. Esta nueva forma de gestión, que comprende una relación armónica con los grupos de interés, pasa a tener un rol protagónico en la dirección de los negocios.

Dado este escenario, el uso de reportes de sostenibilidad como evidencia de implementación de dicha filosofía, resulta necesario. Sin embargo, estos no logran evaluar los beneficios económicos obtenidos sobre la base de esta nueva práctica.

La presente investigación nace de la siguiente interrogante: ¿Qué tan rentable implica ser socialmente responsable y de qué manera se puede analizar esta generación de valor? En consecuencia, el propósito es brindar un acercamiento a la medición financiera de los resultados alcanzados a partir de la gestión de la responsabilidad social.

Palabras clave: Responsabilidad social empresarial, desarrollo sostenible, creación de valor para la empresa, indicadores, sostenibilidad, ratios de sostenibilidad

\section{Introducción}

El desarrollo sostenible se define como la «satisfacción de las necesidades en el presente sin poner en peligro la capacidad de las generaciones futuras para satisfacer sus propias necesidades» (Comisión Mundial sobre el Medio Ambiente y el Desarrollo 1987). Aplicar dicho término a la realidad empresarial sugiere una ges- tión de actividades a partir de un enfoque de negocios sostenible. En este, no solo se persigue el máximo valor económico para el accionista, sino un triple beneficio que complementa la rentabilidad económica con la mejora del bienestar social y la preservación del medioambiente (Schwalb 2005: 49). Esta nueva forma de gestión, que advierte la participación de diversos stakeholders $^{1}$ o grupos de interés en la toma de

1. Stakeholder: las personas, los grupos, las empresas, la comunidad y la sociedad, además de los empleados, proveedores, clientes, suministradores de capitales, en cuanto tienen interés en la existencia y desarrollo de una empresa. 
decisiones, exige una mayor transparencia acerca de la sostenibilidad de las actividades en las organizaciones. Es bajo este contexto que surgen los reportes o memorias de sostenibilidad, documentos que proporcionan información sobre el desempeño económico, social y ambiental de la organización informante.

«[...] La elaboración de una memoria de sostenibilidad comprende la medición, divulgación y rendición de cuentas frente a grupos de interés internos y externos en relación con el desempeño al objetivo del desarrollo sostenible. [Esta] debe ser parte de un proceso más amplio para fijar la estrategia de la organización, implantar los planes de acción, y evaluar los resultados. Asimismo, la elaboración de la memoria permite una evaluación sólida del comportamiento de la organización, y puede servir de base para una mejora continua de los resultados. También sirve como herramienta para afianzar vínculos con los grupos de interés y para obtener aportaciones valiosas a los propios procesos de la organización» (Global Reporting Initiative 2006: 5, 8).

Para llevar a cabo esta investigación, se contó con el apoyo de diversos profesionales expertos en el campo de la responsabilidad social y de empresas de distintos sectores de la economía, que vienen realizando reportes de sostenibilidad en los últimos años. Ante ello, se agradece el apoyo brindado por Dante Pesce, director de la organización no gubernamental (ONG) Vincular de Chile; Elsa del Castillo, profesora de la Universidad del Pacífico; Luis Perera, socio de PriceWaterhouseCoopers y autor de Reporte social: un cuarto estado financiero básico, sobre la dimensión social de las empresas (2003); Álvaro Collas, gerente de Soluciones de Negocios Sostenibles de PriceWaterhouseCoopers y Angélica López, gerente de Enterprise Risk Service de Deloitte. Asimismo, este trabajo fue posible gracias a la colaboración de Alejandro Prieto, subgerente de Imagen y Comunicación de Edegel; Carlo Reyes, gerente de Imagen y Comunicación del Banco Continental y José Salazar, gerente de Responsabilidad Social de Antamina.

\section{Antecedentes}

En 1968 se creó el Club de Roma, organización conformada por científicos, políticos, economistas y asociaciones internacionales, cuya finalidad era la promoción del crecimiento económico estable y sostenible de la humanidad. Luego de esta iniciativa, en 1972, se llevó a cabo la primera Cumbre de la Tierra en la Conferencia sobre el Medio Humano de las Naciones Unidas; y en 1984, se organizó una Comisión Mundial sobre el Medio Ambiente y Desarrollo con el objetivo de integrar el medioambiente y la economía en la toma de decisiones (Comisión Mundial sobre el Medio y el Desarrollo 1987).

Hacia 1987 se formalizó el concepto de desarroIlo sostenible en el "Informe de Brundtland», emitido por la comisión del mismo nombre, entendiéndose este como la «satisfacción de las necesidades en el presente sin poner en peligro la capacidad de las generaciones futuras para satisfacer sus propias necesidades» (Comisión Mundial sobre el Medio Ambiente y el Desarrollo 1987). También cabe señalar que en el año 2002 se reunieron representantes de gobiernos, organizaciones no gubernamentales y empresas en la Conferencia Mundial sobre Desarrollo Sostenible (Johannesburgo), para ratificar la importancia de este elemento en la agenda internacional.

Otro tratado internacional con miras a la protección del medioambiente es el Protocolo de Kyoto, que está vigente desde el año 2005 y propone la disminución en la emisión de gases de efecto invernadero dentro del período 2008-2012. Si bien en principio fue adoptado por 129 países, en la actualidad, 166 países han ratificado el acuerdo (Greenpeace s. f.). Sin embargo, Estados Unidos, un país altamente industrializado, se ha negado a ratificar dicho tratado.

Herramientas de generación de reportes de sostenibilidad

En la última década han surgido distintas herramientas para la generación de reportes o memo- 
rias de sostenibilidad. Entre las más destacadas se encuentran: el Balance Social, la Guía para la elaboración de Memorias de Sostenibilidad del GRI, los indicadores Ethos, el Cuarto Estado Financiero Básico, entre otras.

\section{- El Balance Social}

Es una memoria de sostenibilidad que fue empleada, por primera vez, por la empresa francesa Singer en 1972. En 1977, se hizo obligatoria su publicación para las empresas francesas con más de 750 trabajadores. Durante los siguientes veinte años, este reporte fue adoptado de forma gradual por distintas empresas y mejorado por diversas organizaciones, como Ibase, el Instituto Ethos, entre otras.

\begin{abstract}
«Esta herramienta emplea variables e indicadores. Las variables se refieren a aquellos campos de actividad que pueden variar en su contenido, mientras que los indicadores expresan dicha variación. El Balance Social está organizado en dos áreas: interna y externa. La primera se refiere, únicamente, a la información y evaluación del cumplimiento de la responsabilidad social de la empresa con sus trabajadores (características sociolaborales del personal, servicios sociales de la empresa dirigidos al personal e integración y desarrollo del personal), mientras que en el área externa se refiere a la responsabilidad de la empresa hacia los sectores externos con los cuales la compañía tiene algún tipo de relación (relaciones primarias, relaciones con la comunidad y relaciones con otras instituciones)» (Schwalb y García 2004: 11).
\end{abstract}

- Global Reporting Initiative

El Global Reporting Initiative (GRI) es una organización compuesta por expertos de diver- sos grupos de interés (empresas, trabajadores, ONG, inversionistas, auditores, entre otros) creada en el año 1997, cuya finalidad es proporcionar los lineamientos básicos para la elaboración de memorias de sostenibilidad. Para ello, el GRI introdujo la Guía para la Elaboración de Memorias de Sostenibilidad (2006), que constituye «[...] un marco de trabajo común a nivel mundial, con un lenguaje uniforme y parámetros comunes que sirvan para comunicar de una forma clara y transparente las cuestiones relacionadas con la sostenibilidad.» (Global Reporting Initiative 2006: 4).

El reporte de sostenibilidad propuesto por el GRI plantea una metodología que permite informar sobre los tres pilares del desarrollo sostenible: el desempeño económico, la responsabilidad social y el cuidado del medioambiente. Por su cobertura de información, el GRI se ha convertido en una herramienta de aceptación en el ámbito global. Al año 2007, alrededor de 470 compañías han presentado sus reportes de sostenibilidad, que han sido elaborados sobre la base de dicha metodología, y han sido certificadas.

Esta herramienta de gestión se basa en la aplicación de los principios de materialidad, participación de grupos de interés, contexto de sostenibilidad y exhaustividad para la elaboración de indicadores de desempeño económico, social y ambiental. Asimismo, los indicadores de cada sección se subdividen en indicadores principales (o centrales) e indicadores adicionales, los cuales pueden presentar tanto información cualitativa como cuantitativa. Además, el GRI propone suplementos sectoriales que complementan la memoria de sostenibilidad para trece industrias ${ }^{2}$.

- Indicadores Ethos

El Instituto Ethos es una organización no gubernamental que representa un esfuerzo re-

2. Los suplementos sectoriales se dirigen a: aeropuertos, confecciones y calzado, automotriz, construcción, servicio eléctrico, servicios financieros, procesamiento de alimentos, logística y transportes, minerales y metales, ONG, entidades públicas, telecomunicaciones y operadores turísticos. 
gional (Brasil) para movilizar, sensibilizar y ayudar a las compañías a administrar sus negocios de una manera socialmente responsable. Su principal característica es el interés por establecer patrones éticos para las relaciones con los colaboradores, clientes, proveedores, comunidades, accionistas, entidades públicas y el medioambiente (Instituto Ethos 2004).

Los indicadores Ethos de responsabilidad social son una herramienta correlacionada a los principios del Pacto Global. Estos indicadores pueden ser de profundidad, binarios o cualitativos, los cuales, a la vez, se subdividen en siete aspectos: (1) valores, transparencia y gobernación; (2) público interno; (3) medioambiente; (4) proveedores; (5) consumidores y clientes; (6) comunidad y (7) Gobierno y sociedad.

\section{- Cuarto Estado Financiero Básico}

Es una iniciativa propuesta por Luis Perera, socio de PriceWaterhouseCoopers Chile S.A., en el año 2002, que busca contribuir a la medición de la responsabilidad social empresarial, identificándola según los grupos de interés de las organizaciones.

Esta metodología esboza un modelo de reporte en donde se presenta la información financiera de las diversas actividades de responsabilidad social de la empresa en términos de riqueza o valor generado para la organización, subdivida al mismo tiempo según cada grupo de interés de la empresa: las personas, el Estado, la comunidad, los propietarios o accionistas, la propia empresa y los financiadores de la actividad empresarial.

Como parte complementaria a este estado financiero, se propone que se incluyan notas explicativas sobre políticas de responsabilidad social en la organización en aspectos del gobierno corporativo, código de ética, desarrollo de comunidad, entre otros.

\section{Balanced Scorecard}

El Balanced Scorecard es una herramienta de gestión, introducida por Robert Kaplan y David Norton en 1992, que tiene como fin la medición de los objetivos estratégicos de la organización en función de la visión y los planes de acción. Esta evaluación se realiza sobre la base de indicadores financieros y no financieros de los factores claves que impactarán en los resultados de la organización.

Esta herramienta está organizada en cuatro perspectivas: (1) financiera, (2) clientes, (3) procesos internos y (4) aprendizaje y crecimiento.

En la perspectiva financiera se busca integrar los objetivos financieros con la estrategia de la organización, y engloba a las demás perspectivas. Estos objetivos deben traducirse en indicadores que demuestren la actuación de la empresa en términos económicos, tales como el crecimiento, la rentabilidad, la reducción de costos, entre otros. En cuanto a la perspectiva del cliente, se identifican los segmentos de mercado y los principales clientes a los que la empresa se dirige. Asimismo, se generan indicadores clave para lograr una vinculación sostenible con los mismos. En la perspectiva de los procesos internos, se identifican los procesos más relevantes para cumplir con las metas propuestas en las demás perspectivas, con especial énfasis en la perspectiva financiera y de clientes. Por último, se encuentra la perspectiva de aprendizaje y desarrollo, en donde se buscan objetivos e indicadores que mejoren los procesos de innovación y crecimiento para la organización.

\section{Trabajo de campo}

Selección de indicadores de la guía de elaboración de memorias del GRI

La guía de elaboración de memorias de sostenibilidad del GRI propone una batería de 79 indicadores clasificados en: 9 indicadores para la dimensión económica; 30, para la dimensión ambiental y 40, para la dimensión social. Dentro 
de la dimensión social, estos se subdividen en: 14 para prácticas laborales y ética de trabajo, 9 para derechos humanos, 8 para sociedad y 9 para responsabilidad sobre productos.

Luego de haber realizado un entendimiento general de estos indicadores, se procedió a determinar sus características en términos del tipo de información requerida para su construcción: cua- litativa o cuantitativa. Debido a que el objetivo del estudio es la medición financiera de la gestión de la responsabilidad social, se priorizaron los indicadores cuantitativos para luego establecer el impacto financiero -directo o indirecto- en la gestión de la organización. Bajo estos criterios, se seleccionaron los indicadores de las dimensiones económica, ambiental y social, que se presentan en los cuadros 1,2 y 3 , respectivamente.

\section{Cuadro 1}

\section{Indicadores de la dimensión económica}

\begin{tabular}{|c|l|}
\hline EC1 & $\begin{array}{l}\text { Valor económico directo generado y distribuido, incluyendo ingresos, costos de explotación, retri- } \\
\text { bución a empleados, donaciones y otras inversiones en la comunidad, beneficios no distribuidos y } \\
\text { pagos a proveedores de capital y a gobiernos. }\end{array}$ \\
\hline EC3 & Cobertura de las obligaciones de la organización debidas a programas de beneficios sociales. \\
\hline EC6 & $\begin{array}{l}\text { Política, prácticas y proporción de gasto correspondiente a proveedores locales en lugares donde } \\
\text { se desarrollen operaciones significativas. }\end{array}$ \\
\hline EC8 & $\begin{array}{l}\text { Desarrollo e impacto de las inversiones en infraestructuras y los servicios prestados principalmen- } \\
\text { te para el beneficio público mediante compromisos comerciales, pro-bono, o en especie. }\end{array}$ \\
\hline
\end{tabular}

Fuente: Global Reporting Initiative (2006: 29).

\section{Cuadro 2}

\section{Indicadores de la dimensión ambiental}

\begin{tabular}{|c|c|}
\hline EN1 & Materiales utilizados, por peso o volumen. \\
\hline EN3 & Consumo directo de energía desglosado por fuentes. \\
\hline EN4 & Consumo indirecto de energía desglosado por fuentes primarias. \\
\hline EN5 & Ahorro de energía debido a la conservación y a mejoras en la eficiencia. \\
\hline EN6 & $\begin{array}{l}\text { Iniciativas para proporcionar productos y servicios eficientes en el consumo de energía o basa- } \\
\text { dos en energías renovables, y las reducciones en el consumo de energía como resultado de } \\
\text { dichas iniciativas. }\end{array}$ \\
\hline EN7 & $\begin{array}{l}\text { Iniciativas para reducir el consumo indirecto de energía y las reducciones logradas con dichas } \\
\text { iniciativas. }\end{array}$ \\
\hline EN8 & Captación total de aguas por fuentes. \\
\hline EN10 & Porcentaje y volumen total de agua reciclada y reutilizada. \\
\hline EN22 & Peso total de residuos gestionados, según su tipo y método de tratamiento. \\
\hline EN28 & $\begin{array}{l}\text { Coste de las multas significativas y número de sanciones no monetarias por incumplimiento de } \\
\text { la normativa ambiental. }\end{array}$ \\
\hline
\end{tabular}

Fuente: Global Reporting Initiative (2006: 30-31). 


\section{Cuadro 3}

\section{Indicadores de la dimensión social}

\begin{tabular}{|c|c|}
\hline LA1 & Desglose del colectivo de empleados por tipo de empleo, por contrato y por región. \\
\hline LA2 & $\begin{array}{l}\text { Número total de empleados y rotación media de empleados, desglosados por grupo de edad, } \\
\text { sexo y región. }\end{array}$ \\
\hline LA7 & $\begin{array}{l}\text { Tasas de absentismo, enfermedades profesionales, días perdidos y número de víctimas morta- } \\
\text { les relacionadas con el trabajo por región. }\end{array}$ \\
\hline LA8 & $\begin{array}{l}\text { Programas de educación, formación, asesoramiento, prevención y control de riesgos que se } \\
\text { apliquen a los trabajadores, a sus familias o a los miembros de la comunidad en relación con } \\
\text { enfermedades graves. }\end{array}$ \\
\hline LA10 & Promedio de horas de formación al año por empleado, desglosado por categoría de empleado. \\
\hline S08 & $\begin{array}{l}\text { Valor monetario de sanciones y multas significativas y número total de sanciones no moneta- } \\
\text { rias derivadas del incumplimiento de leyes y regulaciones. }\end{array}$ \\
\hline PR9 & $\begin{array}{l}\text { Costo de aquellas multas significativas fruto del incumplimiento de la normativa en relación } \\
\text { con el suministro y uso de productos y servicios de la organización. }\end{array}$ \\
\hline
\end{tabular}

Fuente: Global Reporting Initiative (2006: 34, 38-39).

\section{Indicadores propuestos}

El modelo de indicadores presentado a continuación se trabajó a partir del análisis integrador de la guía de elaboración de memorias de sostenibilidad del GRI (Global Reporting Initiative 2006) con el Balanced Scorecard. Se observó que ambas perspectivas se podían relacionar mediante el empleo de un enfoque de stakeholders. Por ello, la propuesta de indicadores se desarrolla evaluando el impacto de la gestión de la responsabilidad social en cada uno de los grupos de interés de la organización, los cuales se clasifican en: (1) colaboradores, (2) proveedores, (3) Gobierno, (4) comunidad y (5) medioambiente.

Asimismo, se decidió emplear este enfoque porque, según la investigación realizada por Francisco Durand de la Universidad de Texas de San Antonio, entre el $42 \%$ y $62 \%$ de las empresas socialmente responsables consideran importantes, en el siguiente orden, la preocupación por el medioambiente, seguida por las condiciones laborales de los empleados, la calidad de sus productos y, por último, su relación con los proveedores (Durand 2008: 242).

\section{Colaboradores}

Las organizaciones reconocen la importancia de la gestión del capital humano, pues constituye una ventaja competitiva. «El término de capital humano describe el valor económico del conocimiento, las habilidades y las capacidades de los empleados» (Bohlander y Scott 2008: 14). De esta manera, los indicadores presentados en esta sección relacionan la gestión sostenible de los recursos humanos con los impactos de las actividades y los programas sociales. Esto se refleja en la disminución de la rotación y el ausentismo del personal, el incremento de la productividad laboral, y la salud y seguridad en el trabajo; aspectos mencionados en la perspectiva de aprendizaje y crecimiento del Balanced Scorecard.

\section{- Capacitación a colaboradores}

El objetivo principal de la capacitación es el aprendizaje y crecimiento de los colaboradores, por medio de la adquisición de conocimientos y el desarrollo de habilidades y capacidades. Esta se puede brindar mediante la formación, que consiste en la enseñanza de 
habilidades específicas y/o en la corrección de deficiencias para mejorar, de manera rápida, el rendimiento de los trabajadores. Por otro lado, mediante el desarrollo se busca el enriquecimiento de los recursos humanos en el largo plazo, gracias a la generación de habilidades futuras (Bohlander y Scott 2008: 15).

A continuación, se plantea un conjunto de ratios basados en los indicadores EC1, LA7 y LA10 del GRI para medir el impacto financiero de la capacitación del personal.

a. Capacitación $\begin{gathered}\text { del personal } \\ \text { operativo }\end{gathered}=\frac{\text { Capacitación del personal operativo }}{\text { Gasto de personal operativo }}$

Este indicador relaciona la inversión en capacitación del personal operativo con respecto al gasto de personal operativo. Los métodos de capacitación se clasifican de acuerdo con el perfil del capacitado: empleados no gerenciales y gerentes. Para el cálculo del numerador de este indicador, se consideran los costos para la capacitación de los empleados no gerenciales, específicamente personal operativo, los cuales pueden provenir de capacitaciones en el lugar de trabajo o fuera de él, formación cooperativa, instrucción en el aula, instrucción programada, métodos audiovisuales, aprendizaje electrónico y métodos de simulación (Gómez-Mejía et al. 2001).

El gasto de personal operativo incluye la compensación correspondiente a los servicios prestados por los empleados del área operativa a la empresa. Dentro de estas cargas, se consideran los sueldos, los salarios, las comisiones, las remuneraciones en especie, otras remuneraciones, las vacaciones, la seguridad y la previsión social, y otras cargas asociadas.

b. Costo
unitario de
mano de
obra
El costo unitario de mano de obra se calcula dividiendo el gasto incurrido en el personal operativo entre las unidades producidas por la empresa. Conforme la empresa incremente la capacitación al personal operativo, el costo unitario de mano de obra debería reducirse por la mayor productividad de los empleados, la reducción de pagos al personal por horas extras, entre otros.

c. $\underset{\text { Productos }}{\text { defectuosos }}=\frac{\text { Productos defectuosos }}{\text { Unidades producidas }}$

Este ratio calcula los productos defectuosos debido a los errores cometidos por el personal con respecto al total de unidades producidas. Conforme la empresa invierte en capacitación del personal operativo, el indicador de productos defectuosos debería disminuir.

- Rotación y Ausentismo de personal «La rotación de personal se refiere al movimiento de empleados que abandonan una organización» (Bohlander y Scott 2008: 89). El conjunto de ratios presentados a continuación se construye sobre la base de los indicadores $L A 1$, LA2, EC1 y EC3 del GRI.

$\begin{aligned} & \text { a. } \\ & \text { Retención } \\ & \text { de personal }\end{aligned}=\frac{\text { Programas de retención de personal }}{\text { Gasto de personal }}$

Este indicador calcula la proporción del gasto de personal que ha sido destinado para programas de retención de personal. Este último comprende los diversos planes de incentivos (bonos, pago por méritos, pago por mérito de suma global, premios de incentivos y reconocimientos, incentivos de ventas, planes de incentivos grupales, planes de gainsharing, planes de participación de utilidades, planes de adquisición de acciones por parte de los empleados, entre otros), talleres de planeación de carrera, counseling de carrera, actividades de integración, becas de estudio, entre otros. 
El gasto de personal incluye la compensación correspondiente a los servicios prestados por los empleados del área operativa y no operativa de la empresa. Los componentes considerados son: sueldos, salarios, comisiones, remuneraciones en especie, remuneraciones al directorio, otras remuneraciones, vacaciones, seguridad y previsión social, y otras cargas asociadas.

b. Gastos

$\underset{\substack{\text { a la ruptura } \\ \text { laboral }}}{\text { asociados }}=\frac{\text { Programas de retención de persona }}{\text { Gasto de personal }}$

La ruptura laboral se produce cuando un empleado deja de pertenecer a la organización. Esto puede ocurrir por la eliminación del puesto de trabajo o la sustitución del empleado actual por uno nuevo. De esta manera, el indicador muestra cuánto representa el gasto asociado a la ruptura laboral con respecto al gasto de personal.

Cabe mencionar que los gastos asociados a la ruptura laboral incluyen: (1) los costos de separación, que están conformados por la indemnización por despido, los beneficios sociales (gratificaciones, compensaciones por tiempo de servicio y vacaciones) y otros gastos relacionados con la administración del despido, (las entrevistas para la ruptura y la asistencia para la colocación en el exterior); (2) los costos de reemplazo, que abarcan los costos de reclutamiento (anuncios de puestos vacantes y honorarios de reclutadores profesionales o headhunter) y los costos relacionados con la selección, la contratación y la colocación del nuevo personal (costo de las entrevistas, de las pruebas de conocimiento, psicológicas y médicas, comprobación de referencias, entre otros); y (3) los costos de formación del nuevo empleado; es decir, aquellos que se incurren para la capacitación del nuevo personal en el desempeño de su puesto de trabajo, que pueden ser: costos directos como la instrucción (cursos especializados), compra de libros y materiales, entre otros.
Por otro lado, el denominador considera los gastos de personal descritos en el indicador anterior (retención de personal).

En la medida que la empresa invierta en programas de retención de personal, el indicador de gastos asociados a la ruptura laboral debería disminuir.

c. $\underset{\text { personal }}{\text { Salida de }}=\frac{N^{\circ} \text { salidas de personal }}{\text { Total de personal }}$

El índice de salida de personal se calcula tomando en cuenta el número de bajas voluntarias e involuntarias con respecto al total de personas de la organización (Fitz-Enz 2003). Este indicador, al igual que el de gastos asociados a la ruptura laboral, está inversamente relacionado con la inversión en programas de retención de personal.

d. $\underset{\text { laboral }}{\text { Ausentismo }}=\frac{\text { Horas perdidas }}{\text { Horas trabajadas }}$

El ratio de ausentismo laboral se obtiene por medio de la división de las horas perdidas por ausentismo entre el total de horas trabajadas, y se entiende de manera similar al índice de salida de personal.

e. Costo de ausentismo $=\quad N^{\circ}$ horas ausentismo $\times$ costo

Para poder cuantificar el costo de ausentismo, se multiplica las horas de ausentismo por el costo promedio de personal por hora. Este indicador también muestra una relación inversa con la inversión en retención de personal.

- Salud y Seguridad laboral

La salud y seguridad laboral constituyen una inversión social importante para el bienestar de los empleados y del empleador. Crear un ambiente laboral sano y seguro es esencial, dado que se evitan accidentes y previenen enfermedades que 
constituyen costos potenciales para la empresa. Ello, a su vez, se refleja en la reducción de las tasas de ausentismo, tasas de rotación, entre otros (De Belaunde et al. 2001: 27).

Asimismo, los gobiernos intervienen mediante la promulgación de normas que regulan los estándares laborales ${ }^{3}$, como protección, prevención y capacitación de salud y seguridad, entre otras.

El conjunto de indicadores que se presenta a continuación, se encuentra alineado a los indicadores LA1, LA7, LA8 y EC1 del GRI.

a. Gastos gastos relacionados con la salud $\begin{gathered}\text { promedio de } \\ \text { salud y } \\ \text { seguridad }\end{gathered}=\frac{y \text { seguridad laboral }}{\text { Total de personal }}$

Este indicador determina el gasto promedio de salud y seguridad laboral por empleado, y se obtiene dividiendo el gasto relacionado con la salud y seguridad laboral entre el total de personal en la organización. El numerador se entiende como el gasto destinado a construir un ambiente de seguridad laboral adecuado, tomar las precauciones generales en el ámbito de salud de los trabajadores, la adquisición de equipos de protección personal, la información y formación acerca de peligros en la seguridad, entre otros aspectos.

b. $\underset{\text { accidentado }}{\text { Personal }}=\frac{\text { Personal accidentado }}{\text { Total de personal }}$

Este ratio muestra la proporción del personal accidentado con respecto al total de trabajadores de la empresa. El numerador indica el número de personas que han sufrido siniestros laborales (accidentes, fallecimientos, entre otros).

c. $\begin{gathered}\text { Gastos } \\ \text { promedio de } \\ \text { accidentes }\end{gathered}=\frac{\text { Gastos de personal accidentado }}{\text { Total de personal }}$
Mediante este indicador se trata de medir el costo promedio de accidentes, por lo que se debe determinar los costos relacionados con los accidentes de los empleados, los cuales varían de acuerdo con la gravedad del incidente ocurrido. Cabe mencionar que el personal accidentado puede retornar a sus labores una vez recuperado, quedar inválido permanentemente o fallecer. En el primer caso, se debe incluir el costo promedio de unidades no producidas por el accidente y el costo promedio del salario del empleado. El segundo caso implica una indemnización que contempla la prestación por incapacidad permanente, por lesiones, por cobertura médica y rehabilitación, entre otras. Finalmente, si el personal no sobrevive, se debe considerar los gastos de sepelio e indemnizaciones a los familiares. Luego, se calcula el costo total por accidentes, el cual se dividirá entre el total de empleados.

En la medida que el gasto promedio de salud y seguridad se incremente, el nivel de accidentes debería disminuir, por lo que el indicador de costo de accidente promedio también se reduciría.

\section{Proveedores}

Una de las dimensiones de la responsabilidad social empresarial comprende la gestión de las relaciones con los proveedores.

«Estos son las personas o entidades encargadas
de suministrar las materias primas, servicios o
productos terminados (mercadería) necesarios
para que la empresa pueda desarrollar su activi-
dad normal. Adicionalmente, los proveedores ayu-
dan en parte a financiar los inventarios, permi-
ten presentar novedades a los clientes, asesoran
en la comercialización de los productos, com-
parten información sobre participación, tenden-
cias y cambios de mercado» (Montoya 2002: 51 ).

3. En el caso peruano se encuentra vigente el Reglamento de Seguridad y Trabajo (D.S. N 009-2005 TR). 
Este grupo de interés conforma un eslabón elemental dentro de la cadena de valor, pues los proveedores también pueden convertirse en una ventaja competitiva para la empresa. Una adecuada selección, desarrollo y colaboración con el proveedor puede resultar en una oportunidad para la mejora de la calidad de los insumos, el incremento de la eficiencia en entregas y, por tanto, en un mayor control de existencias, acceso a menores precios que permitan el ahorro de costos, entre otros beneficios. De esta manera, las prácticas y los programas de responsabilidad social deberían incluir el desarrollo de relaciones sostenibles con los proveedores, lo cual se desarrolla en la perspectiva de los procesos internos del Balanced Scorecard mediante los costos operativos, la eficiencia y el rendimiento de insumos.

\section{- Capacitación a proveedores}

La capacitación a proveedores es una práctica empleada por las organizaciones para mejorar la gestión de la cadena de abastecimiento. Esta puede realizarse por medio de capacitadores externos (contratación de terceros) y/o internos (funcionarios de la empresa), con el fin de mejorar los tiempos de entrega, la calidad, los precios y servicios asociados.

El conjunto de indicadores presentados en esta sección están basados en los indicadores EC1 y EC6 del GRI.

a. Capacitación a proveedores $=\frac{\text { Capacitación a proveedores }}{\text { Compras }}$

El ratio determina la proporción de las compras que se destina a la capacitación de los proveedores y se calcula dividiendo el gasto de capacitación a los proveedores entre las compras. El primer componente incluye el costo de contratación de servicios de terceros y/o el costo de las horas destinadas por el personal interno a la capacitación.

«Las compras representan las adquisiciones relacionadas con bienes de cambio, en las cuales solo se deberá contabilizar la compra de bienes que se adquieren para ser destinados a la venta o pera ser consumidos en la producción directa o indirectamente. Las compras incluyen mercaderías, materias primas y auxiliares, envases y embalajes, suministros diversos y gastos diversos vinculados con las compras» (Estudio Caballero Bustamante 2007: 13).

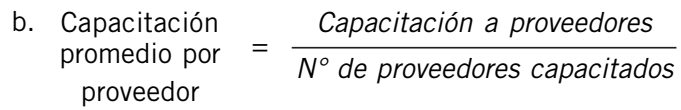

Este indicador permite observar la evolución del gasto en capacitación promedio por proveedor capacitado.

c. Costo unitario de producción Costo de producción Unidades producidas

El costo unitario de producción se determina dividiendo los costos de producción entre la cantidad de unidades producidas. Los costos de producción incluyen los costos incurridos en el consumo de materiales directos, mano de obra y otros costos indirectos de fabricación.

Este indicador se vincula inversamente con la capacitación a proveedores, en el sentido que a mayor inversión en capacitación a proveedores, los costos de producción deberían disminuir por el mayor rendimiento del insumo, mayor eficiencia en el manejo de inventarios, entre otros.

d. Devoluciones $=\frac{\text { Devoluciones }}{\text { Compras }}$

Las devoluciones sobre compras pueden darse por exceso, averías, cambios de presentación y precios. Estas deben considerarse como numerador del ratio presentado en términos monetarios. Por otro lado, el denominador considera las compras ya descritas en el primer indicador (capacitación a proveedores). 
En la medida que la empresa invierta en capacitación a los proveedores, las devoluciones se deberían reducir en el tiempo.

\section{Gobierno}

El ratio de multas sobre ventas se alinea con los indicadores EC1, EN28, S08 y PR9 del GRI, y manifiesta el nivel de incumplimiento de las normas ambientales, sociales y legales con respecto al ingreso total de la empresa.

a. Multas $=\frac{\text { Multas }}{\text { Ventas }}$

Las multas incluyen las sanciones monetarias por: incumplimiento de la normativa ambiental (como obligaciones de limpieza ambiental $\mathrm{u}$ otras responsabilidades medioambientales onerosas), incumplimiento de las leyes y regulaciones (como fraude contable, discriminación en el puesto de trabajo, corrupción, entre otros) e incumplimiento de la normativa en relación con el suministro y el uso de productos y servicios de la organización. Las ventas, por otro lado, incluyen las ventas netas realizadas.

Las empresas responsables deberían haber superado la barrera normativa, por lo que este ratio debería tender a cero.

b. $\begin{aligned} & \text { Donaciones } \\ & \text { deducibles }\end{aligned}=\frac{\text { Donaciones deducibles }}{\text { Donaciones }}$

El indicador calcula la proporción de donaciones deducibles con respecto al total de donaciones realizadas. En términos económicos, las empresas buscan que este indicador sea igual a uno, porque de esta manera la totalidad de las donaciones podría ser deducible de la base imponible.

\section{Comunidad}

Las organizaciones deben considerar no solo el entorno en el que operan al tomar sus decisiones, sino también deben ser partícipes del desarrollo local. Este se refiere al proceso de mejora de la calidad de vida en la población asentada en un ámbito geográfico determinado. Así, la mejora puede darse por el incremento sostenido de la actividad económica local, la satisfacción de las necesidades fundamentales de la población cercana, el desarrollo de un sistema de información, manejo y control adecuado del medio ambiente, entre otros (Caravedo 1999: 49).

La guía de elaboración de memorias de sostenibilidad del GRI muestra los riesgos de las interacciones comunitarias y los impactos que tienen las operaciones de las compañías en las comunidades en las que operan, los cuales se miden mediante los indicadores EC1, EC6, EC8, LA8 y SO1.

a. $\begin{gathered}\text { Paros y } \\ \begin{array}{c}\text { levantamientos la } \\ \text { de la } \\ \text { comunidad }\end{array}\end{gathered}=\frac{\begin{array}{c}\text { Costos de paros y levantamientos } \\ \text { de la comunidad }\end{array}}{\text { Ventas }}$

Los paros y levantamientos suspenden la producción de la empresa, por lo que se incurre en costos hundidos, expresados en número de unidades no producidas por el costo unitario de producción.

b. $\underset{\text { comunitaria }}{\text { Infraestructura }}=\frac{\text { Infraestructura comunitaria }}{\text { Ventas }}$

Este indicador mide la relación entre la inversión en infraestructura para la comunidad con respecto a las ventas de la compañía. El numerador comprende: construcción de vías terrestres (carreteras); instalación de servicios eléctricos, de agua y desagüe; construcción de centros de salud, escuelas, centros culturales y de entretenimiento; entre otros.

c. $\begin{gathered}\text { Compra a } \\ \text { proveedores } \\ \text { locales }\end{gathered}=\frac{\text { Compra a proveedores locales }}{\text { Compras }}$

Las capacitaciones a la comunidad en labores productivas pueden beneficiar tanto a los proveedores como a la empresa. Los primeros pueden convertirse en proveedores locales y/o 
mejorar su calidad de vida; mientras que la empresa puede adquirir diversos insumos a menores costos, dadas las ventajas de los proveedores locales. Estas se ven reflejadas, principalmente, en el menor costo logístico y de mano de obra.

Conforme el ratio de compras a proveedores locales con respecto a las compras totales se incremente, se espera que la empresa obtenga beneficios por reducción de costos.

\section{Medioambiente}

La dimensión medioambiental es una de las más importantes en la gestión de la responsabilidad social. Esta comprende los sistemas naturales que condicionan la vida de las personas o de la sociedad y el sostenimiento de la empresa a lo largo del tiempo. Específicamente, la relación que tienen las organizaciones con el medioambiente se refiere al consumo de los recursos que lo componen, a las emisiones que puedan afectarlo y al impacto que puedan tener los productos o servicios brindados en el ambiente.

El desarrollo de nuevas tecnologías se ha enfocado a producir maquinaria que optimice el uso de recursos, que reduzca los tiempos de ciclo y que sea amigable con el medioambiente. Además, existe una preocupación latente por encontrar nuevas fuentes de energía, más económicas y cuyas emisiones sean menos contaminantes. De esta manera, la empresa busca beneficiarse por ahorros en el nivel productivo, por una mejor imagen en el ámbito corporativo y, en algunos casos, por posibles beneficios futuros (bonos de carbono $)^{4}$. Esto también se relaciona con la perspectiva de aprendizaje y crecimiento, así como de procesos internos, del Balanced Scorecard.
- Reutilización del agua

El recurso hídrico puede ser suministrado por fuente natural y/o por alguna empresa prestadora de este servicio. El costo del agua procedente de la primera fuente comprende el costo de tratamiento (eliminación de sólidos, arena y sustancias grasas) cada vez que sea utilizada; mientras que el de la segunda fuente comprende, además de lo mencionado anteriormente, el costo de suministro.

El conjunto de ratios se encuentra alineado a los indicadores EN8 y EN10 del GRI y tiene como objetivo medir el ahorro de costos por consumo de agua en el proceso productivo por la reutilización de dicho recurso.

$\underset{\text { a. }}{\stackrel{\text { Reutilización }}{\text { de agua }}}=\frac{\text { Volumen de agua reutilizada }}{\text { Volumen de agua consumida }}$

En primer lugar, se debe calcular el volumen de agua reutilizada en metros cúbicos. Se debe tomar en cuenta que el agua provista por fuente natural es calificada como reutilizada desde la primera vez que es empleada en el proceso productivo; mientras que la adquirida en el mercado es calificada así desde la segunda vez.

En segundo lugar, se calcula el total de agua consumida en el proceso productivo en metros cúbicos. Esta información se obtiene de las fuentes primarias utilizadas y del sistema de inventario de agua. Al considerar solo el agua consumida en el proceso productivo, al total de agua percibida se le debe restar aquella que aún no ha pasado a formar parte del proceso productivo (inventario de agua).

Con respecto a la lectura del indicador, se estima que conforme el porcentaje de agua reutilizada vaya creciendo, el costo del agua por unidad producida será menor. Esto se ex-

4. La reutilización de energía es importante porque la inversión en maquinaria especializada se recuperará al no recurrir a un proveedor externo y al mejorar la posición, dadas las posibilidades de una emisión de bonos de carbono. Esta emisión daría la opción de recuperar la inversión en el corto plazo y se tendría que incurrir en costos de certificación, emisión y monitoreo, los cuales, en vista de la magnitud de la operación, no serán significativos (Sociedad Nacional de Minería, Petróleo y Energía 2008). 
plicará más adelante, mediante la exposición de los siguientes indicadores, en los cuáles se incluye el costo de reutilización, el costo total del agua consumida, entre otros.

b. Costo unitario $\underset{\text { de agua }}{\text { reutilizada }}=\frac{\text { Costo de agua reutilizada }}{\text { Unidades producidas }}$

El numerador de este indicador se puede calcular mediante la sumatoria del costo de las actividades correspondientes al tratamiento del recurso. Estas pueden ser las siguientes: la depreciación de la maquinaria, el costo de mantenimiento de la maquinaria, el consumo de energía de la misma, la depreciación de los metros cuadrados de la instalación en donde se coloca el equipo, el costo de supervisión (considerado como mano de obra indirecta) y el costo de los insumos necesarios para el tratamiento del agua. Por otro lado, se debe calcular el número de unidades producidas.

c. Costo unitario

de agua comprada y $=$ utilizada

$\begin{gathered}\text { Costo de agua comprada } \\ \text { y utilizada }\end{gathered}$
Unidades producidas

Como se explicó anteriormente, al costo del agua suministrada por la empresa prestadora del servicio público se le debe restar el costo de dicho recurso almacenado (inventario de agua). Se estima que el costo unitario de agua reutilizada debe ser inferior al costo unitario de agua comprada y utilizada.

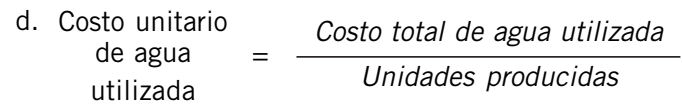

Para determinar el costo unitario del agua usada se debe hallar el costo total de agua utilizada en el proceso productivo, de la misma manera que se ha calculado el costo del agua reutilizada (indicador $b$ ), y se le debe agregar el costo del agua comprada y utilizada (indica- dor c). Luego, este importe se divide entre las unidades producidas

Este monto se debe comparar con el costo de agua reutilizada por unidad producida, y con el costo de agua comprada y utilizada por unidad producida.

El costo total de agua utilizada también se puede hallar considerando un promedio ponderado de ambos tipos de agua usada en el proceso productivo. Se estima que la relación entre la reutilización de agua y el costo unitario de agua usada es inversamente proporcional.

\section{- Eficiencia energética}

La energía consumida consta de tres componentes: la energía primaria, la energía secundaria y la energía útil. El primero comprende los insumos provenientes de fuentes naturales que pueden ser utilizados de manera directa; el segundo, los insumos transformados a partir de la energía primaria; y el tercero consiste en la energía que realmente se consume, sin considerar la energía que se pierde en los equipos de aprovechamiento energético (Ministerio de Energía y Minas 1999).

Con respecto a la energía primaria, se reconocen dos clases de recursos: los renovables y los no renovables. Dentro del primer grupo se encuentran los biocombustibles, el etanol y el hidrógeno; mientras que en el segundo grupo se ubican el carbón, el gas natural y los combustibles destilados del petróleo crudo.

Por otro lado, los recursos de la energía secundaria, al proceder de la energía primaria, también se clasifican en fuentes renovables y no renovables. En el primer grupo se incluye la energía generada a partir del uso del viento (eólica), de las radiaciones energéticas (solar), de la descomposición de desperdicios y/o desechos orgánicos (biomasa), de la caída de agua (hidráulica), de cuerpos que tengan calor (calórica) y del movimiento molecular (térmica); 
mientras que en el segundo grupo, la energía generada a partir del consumo por combustión (química), de almacenamiento en el campo eléctrico de un condensador (eléctrica), entre otras (Ministerio de Energía y Minas 1999).

El uso eficiente de los recursos correspondientes a la energía primaria y, en consecuencia a la energía secundaria, es primordial en la operación de la empresa para la reducción de costos y para disminuir el impacto ambiental. Dada esta oportunidad, las organizaciones optan por el cambio de fuente energética, tomando en cuenta los precios de mercado y la inversión necesaria. Además, existen formas alternativas para reducir los costos de uso de energía, como reparar fugas, instalar sistemas de retorno, entre otras.

Los siguientes ratios buscan medir el ahorro de costos como consecuencia de la eficiencia energética. Estos están basados en los indicadores EN3, EN4, EN5, EN6 y EN7 del GRI.

a. Consumo de energía por fuente Consumo de energía clasificado $=\frac{\text { por fuente }}{\text { Consumo total de energía }}$

Para empezar, se debe reconocer la proporción de consumo de energía por cada fuente. Esta información se debe uniformizar en una unidad de medida, gigajulios (GJ), lo que permitirá una evaluación de las fuentes y de los costos unitarios de cada GJ por fuente energética.

b. Costo de $\begin{gathered}\text { Costo de } \\ \text { energía por } \\ \text { fuente }\end{gathered}=\frac{\text { Costo de energía por fuente }}{\text { Consumo de energía }}$
Luego, se procede a la evaluación del costo total de consumo de energía por fuente con respecto al costo total de energía en el área productiva. Esto se complementa con el indicador anterior, en el sentido de que existe la posibilidad de que se consuma mayor número de GJ por una fuente. Sin embargo, el costo unitario del GJ de la fuente puede ser muy inferior al costo promedio ponderado por GJ consumido.

c. Costo unitario $\begin{aligned} & \text { de energía } \\ & \text { reutilizada }\end{aligned}=\frac{\text { Costo de energía reutilizada }}{\text { Unidades producidas }}$

Cabe mencionar la recuperación de energía debido a su reutilización. La tecnología ha permitido que, hasta el momento, se pueda reutilizar el vapor para la generación de energía calórica mediante un sistema combinado ${ }^{5}$. La reutilización de este disminuye la presión ejercida sobre los recursos del medioambiente $y$, al mismo tiempo, conduce a ahorros significativos por el no consumo de otras fuentes de energía primaria.

Por otro lado, se puede considerar dentro del numerador de este indicador a la energía obtenida sin ningún costo (de la naturaleza), tales como la energía solar, hidráulica, eólica y calórica. Para conseguirla, es necesario contar con instalaciones especiales que puedan captar la energía, almacenarla y transformarla para su utilización en el proceso productivo.

El costo de la energía reutilizada incluye la depreciación de la maquinaria y del espacio destinado a esta, el costo de su mantenimiento, el consumo de otras fuentes de energía (o de la misma), y el costo de la supervisión y/o mane-

5. En la actualidad, se observa que el sistema combinado es utilizado por sectores como el energético (Endesa España, mediante Edegel S.A.A. - Perú), la industria cervecera (Cervecería Nacional S.A. - Panamá) y la industria automotriz (KERS, kinetic energy recovery systems, sistema diseñado y desarrollado por Xtrac, Torotrak y Flybrid Systems que entró en funcionamiento en el año 2009 en las competiciones automovilísticas). Según opiniones recogidas, en el Perú aún no hay motivación para la implementación de sistemas combinados porque si bien se ha optado por el gas como fuente energética, este es muy barato en el mercado, lo que hace que el período de recuperación de la inversión se haga más largo (Sociedad Nacional de Minería, Petróleo y Energía 2005). 
jo del equipo. Una vez calculado el costo de la energía reutilizada por unidad producida, se debe comparar dicho monto con el costo de energía utilizada por unidad producida y con el costo unitario de energía suministrada por empresas de servicio público.

d. Costo unitario de energía $=\frac{\text { Costo de energía }}{\text { Unidades producidas }}$

El costo unitario de energía considera el costo de la energía reutilizada y el costo de energía provista por una empresa prestadora de servicios, divididos entre las unidades producidas.

En la medida que se reconozcan las fuentes de energía utilizadas y su respectivo costo, se podrá emplear dicha información para mejorar la eficiencia en el uso del recurso. De esto, se puede observar una relación inversa entre el costo unitario de energía y el costo unitario de energía reutilizada.

- Tecnología limpia

A continuación se presentan los indicadores que tratan de medir el impacto del ahorro por utilización de nuevas tecnologías, los que se basan en los indicadores EN5, EN6 y EN7 del GRI.

$\begin{gathered}\text { a. Mantenimiento } \\ \text { reparación y } \\ \text { respuestos }\end{gathered}=\frac{\begin{array}{c}\text { Mantenimiento, reparación y } \\ \text { repuestos de maquinarias }\end{array}}{\text { Costo de producción }}$

En primer lugar, se calcula el importe del mantenimiento, reparación y repuestos de las maquinarias; el cual se obtiene del sistema de información contable, mediante el análisis de gastos operativos. Por otro lado, el costo de producción se obtiene del estado de costos de productos terminados y vendidos con la finalidad de determinar la proporción correspondiente al mantenimiento, reparación y repuestos de maquinarias referida a los costos de producción. b. $\begin{gathered}\text { Productos } \\ \text { defectuosos }\end{gathered}=\frac{N^{\circ} \text { de productos defectuosos }}{\text { Unidades producidads }}$

Luego, se determina el ratio de productos defectuosos correspondientes al total de unidades producidas. Para esto, se conocen los productos defectuosos por alguna avería y/o defecto en la maquinaria utilizada en la producción. La cantidad de unidades producidas ha sido recogida en el indicador anterior.

c. $\underset{\text { máquina }}{\text { Horas }}=\frac{\text { Horas máquina de producción }}{\text { Unidades producidas }}$

Este indicador contempla el total de horas máquinas efectivas en la producción, sin considerar las horas de mantenimiento o reparación. En primer lugar, se necesita determinar de manera específica la maquinaria utilizada para dicho fin y tener un control estricto de las horas que no se utilizan por efectos de averías o interrupción del proceso productivo y de las horas destinadas al mantenimiento, tanto preventivo como correctivo. Al total de horas calculadas en el período, se le debe restar las horas mencionadas anteriormente y luego, dividir la diferencia entre las unidades producidas.

d. Consumo de energía

Costo de energía

Unidades producidas

Además, se debe identificar el consumo de energía (en GJ) de las máquinas involucradas en el proceso productivo. Con esto, se podrá reconocer el consumo de energía por unidad producida. Cabe mencionar que la energía utilizada en el proceso productivo incluye la energía empleada tanto en las unidades producidas en buenas condiciones como en los productos que resultaron defectuosos.

Estos indicadores impactan de manera directa e indirecta en los costos de producción, pues se espera que conforme el costo de manteni- 
miento, reparaciones y repuestos con respecto al costo de producción (a) aumente, los productos defectuosos (b), las horas máquina requeridas en producción (c) y la energía requerida (d) disminuyan.

\section{Conclusiones}

- La responsabilidad social, práctica que involucra la participación activa de todos los stakeholders para lograr una gestión sostenible, cada vez es más implementada en el nivel empresarial. Ello se debe a la mayor exigencia del mercado, la existencia de fuentes de regulación internacionales e incluso, a la misma política de las grandes empresas multinacionales.

- Las empresas que tienen conocimiento sobre la responsabilidad social no necesariamente la practican en todos sus aspectos. Hay quienes consideran que esta filosofía se limita a la filantropía; mientras que otros sostienen que la responsabilidad social es una inversión cuyos retornos no son cuantificables en el tiempo.

- Las empresas que incorporan prácticas de responsabilidad social en la gestión consideran que, hasta el momento, estas brindan beneficios intangibles. La mejora de la reputación e imagen corporativa y la comunicación e interacción con diversos stakeholders son el reflejo de algunos de estos beneficios. Por otro lado, son pocos quienes consideran que la responsabilidad social impacta de manera positiva en la gestión de la empresa en el largo plazo, y que pueda ser vista en términos de ahorro en costos y mitigaciones de riesgos.

- La elaboración de reportes de sostenibilidad es poco práctica en el ámbito empresarial. Ello se debe a la falta de una cultura de rendición de cuentas (accountability), ausencia de recursos económicos, falta de sistemas de información que faciliten la identificación de las actividades de la responsabilidad social y el recojo de la información relacionada con esta, entre otros.
- Existen organizaciones que proponen diversas metodologías para la elaboración de reportes de sostenibilidad, como el balance social, la guía de elaboración de memorias de sostenibilidad GRI, los indicadores Ethos y diversas iniciativas en el nivel regional. Cada una de ellas proporciona lineamientos e indicadores distintos que convergen en un mismo objetivo: mostrarse a los stakeholders de una manera transparente.

- Tanto los expertos como las empresas opinan que la metodología para la elaboración de reportes de sostenibilidad, por utilizar en los próximos años, será la propuesta por el GRI, pues esta integra las tres dimensiones del desarrollo sostenible y constituye un lenguaje común que permite la comparación de la información.

- Entre los beneficios del reporte de sostenibilidad propuesto por el GRI se encuentran: el benchmark de la gestión de la responsabilidad social en el tiempo, el diálogo con los diversos grupos de interés, la integración de la perspectiva sostenible a la gestión cotidiana de la organización, la identificación de aspectos de mejora, entre otros.

- La elaboración de memorias de sostenibilidad de acuerdo con el modelo planteado por el GRI presenta dificultades, como la complejidad de algunos indicadores, la no aplicabilidad de estos a ciertas industrias, y la demanda de recursos humanos, económicos y tiempo en la recopilación y procesamiento de la información.

- La metodología propuesta desarrolla indicadores basados en el enfoque de stakeholders: colaboradores, proveedores, Gobierno, comunidad y medioambiente.

- La inversión en programas de responsabilidad social para los colaboradores presenta un retorno económico en términos de reducción de rotación y ausentismo de personal; así como, la disminución de accidentes y mejora de productividad. 
- La capacitación de proveedores permite lograr ahorro en los costos por la reducción de tiempos de entrega, devoluciones y productos defectuosos.

- Los programas para la comunidad, como la mejora de infraestructura local, permiten mitigar riesgos de paros y levantamientos que repercuten en las operaciones normales de la empresa. A su vez, la integración de los pobladores como proveedores de la empresa se traduce en ahorro de costos para la organización.

- Con respecto al medioambiente, la empresa puede invertir en programas de reutilización de agua, eficiencia de consumo energético y tecnología limpia. Esto impacta en la estructura de costos de la compañía y el cuidado del ecosistema.
- Para poder entender la gestión de la responsabilidad de manera integral, se debe tener en cuenta que los indicadores planteados de los distintos stakeholders se interrelacionan y que su interpretación se debe hacer de manera conjunta.

- Las organizaciones deben realizar una evaluación mediante los indicadores propuestos para identificar aspectos de mejora e implementar planes de acción. Esta evaluación se debe llevar a cabo de manera constante para efectos de comparación con períodos anteriores. Cabe mencionar que esto se logrará con el esfuerzo conjunto de los stakeholders y de la empresa. 


\section{Referencias}

ACCOUNTABILITY - INSTITUTE OF SOCIAL AND ETHICAL ACCOUNTABILITY

2003 Norma sobre aseguramiento AA1000. Fecha de consulta: 24/09/2008. <http://www. accountability21.net/uploadedFiles/Resources/ AA1000\%20traduccion\%20marzo\%202006.pdfs.

ANTAMINA

2006 Reporte de Sostenibilidad Antamina 2006. Fecha de consulta: 24/10/2008. <http:// www.antamina.com/boletin/zips/rds_2006.pdf >.

BANCO BILBAO VIZCAYA ARGENTARIA - BBVA

s. f. Información complementaria al informe anual de responsabilidad corporativa 2007. Fecha de consulta: 01/10/2008. <http://www.bbva.com/ TLBB/fbin/InfCompl\%20IARC2007_tcm12161111.pdf\#tcm:12-161887-64>.

BOHLANDER, G. y S. SCOTT

2008 Administración de recursos humanos. 14ª ed. México: Thompson.

CARAVEDO, B.

1999 Lo social y la empresa a fines de siglo. Responsabilidad social empresarial: avances y logros. Lima, Perú: Centro de Investigación de la Universidad del Pacífico, Perú 2021 y SASE.

COMISIÓN MUNDIAL SOBRE EL MEDIO Y EL DESARROLLO

1987 Declaración de Tokio 1987. Ginebra: Comisión Mundial sobre el Medio y el Desarrollo. $<$ http://www.infomedioambiente.es/database/ legislacion/ACUERDOS\%20Y\%20 DECLARACIONES/2.pdf $>$.

\section{COMISIÓN MUNDIAL SOBRE MEDIO AMBIENTE Y} DESARROLLO

1987 Nuestro futuro común. Madrid. España: Alianza editorial.

DE BELAUNDE, J.; B. PARODI y D. MUÑOZ

2001 Cómo promover la responsabilidad social empresarial en el Perú: marco legal e institucional. Lima, Perú: Centro de Investigación de la Universidad del Pacífico.
DURAND, F.

2008 «Empresa y responsabilidad social empresarial: el caso peruano». En: SANBORN, C. y F. PORTOCARRERO S. (eds.). Filantropía y cambio social en América Latina. Lima, Perú: Centro de Investigación de la Universidad del Pacífico y Harvard University, pp. 233-264.

\section{EDEGEL}

2007 Memoria anual - Informe de sostenibilidad 2007. 3 t. Fecha de consulta: 14/10/2008. <http://www.edegel.com/memoria2007/ index.htm>.

\section{ESTUDIO CABALLERO BUSTAMANTE}

2007 Dinámica de las cuentas del estado de ganancias y pérdidas: ingresos y gastos. Lima, Perú: Estudio Caballero Bustamante.

\section{GLOBAL REPORTING INITIATIVE - GRI}

2006 Guía para la elaboración de Memorias de Sostenibilidad 2000-2006. Holanda: Global Reporting Initiative. <http://www.global reporting.org/NR/rdonlyres/415F297B-52894160 - 8 B 6 C - 7 C C 034 D 5 B E 5 2/ 0 / G3_GuidelinesESP.pdf>.

GÓMEZ-MEJÍA, L.; D. BALKIN y R. CARDY

2001 Dirección y gestión de recursos humanos. México: Prentice Hall.

\section{GREENPEACE}

s. f. «Protocolo de Kioto». En: Greenpeace España. Fecha de consulta: 01/09/2008. <http:// www.greenpeace.org/espana/Global/espana/ report/cambio_climatico/protocolo-de-kioto.pdf>.

FITZ-ENZ, J.

2003 El ROI (rendimiento de la inversión) del capital humano. Cómo medir el valor económico del rendimiento del personal. Barcelona, España: Ediciones Deusto.

INSTITUTO ETHOS DE EMPRESAS Y RESPONSABILIDAD SOCIAL

2004 Indicadores Ethos de responsabilidad social empresarial. Fecha de consulta: 22/09/2008. <http://www.ethos.org.br/_Uniethos/ documents/indicadores_2004_espanhol.pdf>. 


\section{MINISTERIO DE ENERGÍA Y MINAS}

1999 Uso racional de energía: eficiencia energética y energías renovables: manual para consultores y expertos. Lima: C. Duisberg Gesellschaft (Alemania) - Proyecto para Ahorro de Energía [del] Ministerio de Energía y Minas.

MONTOYA, A.

2002 Conceptos modernos de administración de compras. Bogotá: Norma.

\section{ORGANIZACIÓN DE COOPERACIÓN Y DESARROLLO ECONÓMICO - OCDE}

2000 Líneas directrices de la OCDE para empresas multinacionales. Fecha de consulta: 13/09/ 2008. <http://www.oecd.org/dataoecd/21/20/ 16975360.pdf>.

\section{PERERA, L.}

2003 Reporte social: un cuarto estado financiero básico, sobre la dimensión social de las empresas. Chile: PricewaterhouseCoopers.

SCHWALB, M.

2005 «Una definición comprehensiva de la responsabilidad social empresarial». En: Punto de Equilibrio, $N^{\circ} 89$, pp. 48-49.

SCHWALB, M. y E. GARCÍA

2004 Instrumentos y normas para evaluar y medir la responsabilidad social empresarial. Lima, Perú: Centro de Investigación de la Universidad del Pacífico.

SOCIEDAD NACIONAL DE MINERÍA, PETRÓLEO Y ENERGÍA - SNMPE

2008 Informe quincenal de la snmpe, $N^{\circ} 69$, setiembre. Lima, Perú: SNMPE, Sector Multisectoriales. <http://www.snmpe.org.pe/ pdfs/Informe_Quincenal/Multisectorial/EEESIQ-69-2008-JL.pdf>.
2005 Informe quincenal de la snmpe, № 2, enero. Lima, Perú: SNMPE, Sector Multisectoriales.

THE LEON H. SULLIVAN FOUNDATION

s. f. The Global Sullivan Principles of Social Responsibility. 03/09/2008. <http:// www.thesullivanfoundation.org/about/ global_sullivan_principles>.

\section{Páginas web}

\section{Corporate Register}

Fecha de consulta: 14/10/2008.

$<$ http://www.corporateregister.com/>.

Dow Jones Sustainability Indexes

Fecha de consulta: 19/09/2008.

$<$ http://www.sustainability-index.com/>.

FTSE The Index Company

Fecha de consulta: 19/09/2008.

$<$ http://www.ftse.com/>.

KLD Research \& Analytics, Inc.

Fecha de consulta: 19/09/2008.

<http://www.kld.com/>.

Organization of Standarization - ISO

Fecha de consulta: 15/09/2008.

$<$ http://www.iso.org/>.

Pacto Global

Fecha de consulta: 01/09/2008.

<http://www.pactoglobal.org>.

Vincular - Responsabilidad Social y Desarrollo Sostenible

Fecha de consulta: 30/09/2008.

$<$ http://vincular.cl/>. 